\title{
Unifying Models for Multiphase Coupled Inductors
}

\author{
Minjie Chen, Senior Member, IEEE
}

\begin{abstract}
This paper presents a unified model for multiphase coupled inductors. By summarizing and comparing three different ways of modeling multiphase coupled inductors, including a) an inductance matrix model derived based on self and mutual inductances; b) a multiwinding transformer model derived based on magnetizing and leakage inductances; and c) an inductance dual model derived based on the inverse of the magnetic reluctances. Critical modeling principles and design insights are reviewed, summarized, and compared. The design equations linking the three models are clarified and correlated. The analysis and comparison are rigorously verified through theoretical verifications and SPICE simulations. The three different models represent identical magnetic coupling relationships in the multiphase coupled inductors, but reveal different fundamental principles and offer distinct design insights.
\end{abstract}

Index Terms-lumped circuit model, multiphase coupled inductor, multiphase buck converter, inductance matrix, reluctance model, inductance dual model.

\section{INTRODUCTION}

$\mathbf{M}$ ULTIPHASE coupled inductor buck converters are widely used in high current voltage regulation module (VRM) applications. Using coupled inductors in multiphase buck converters can improve the efficiency, reduce the flux, improve the transient response, and reduce the output capacitance. Designing high performance multiphase coupled inductor buck converters need good lumped circuit models and design equations [1]-[9].

There are two commonly used models for multiphase coupled inductors: b) an inductance matrix model derived based on self and mutual inductances of the inductance matrix [10][15]; b) a multiwinding transformer model derived based on magnetizing and leakage inductances [16]-[23]. The two models capture identical equations in the electromagnetic domain, but represent different physical interpretation and offers different design insights. Using the inductance matrix model, one will need to convert the magnetic geometry information into the inductance matrix, then apply the matrix to circuit analysis and simulations. Intricate experimental measurements are sometimes needed to identify the inductance matrix. Due to the sophisticated coupling relationship, the complexity of a multiwinding transformer model grows rapidly as the number of windings increases.

A more recently developed method, inductance dual model, which is a dual of a traditional reluctance model, offers complementary advantages to the two forementioned models.

Minjie Chen is with the Department of Electrical Engineering and Andlinger Center for Energy and the Environment at Princeton University, Princeton, NJ 08540, USA.

Charles R. Sullivan is with the Thayer School of Engineering of Dartmouth College, Hanover, NH 03755, USA.

Fred C. Lee is with the Bradley Department of Electrical Engineering and Computer Engineering of Virginia Tech, Blacksburg, VA 24061, USA.

This work was supported by National Science Foundation.
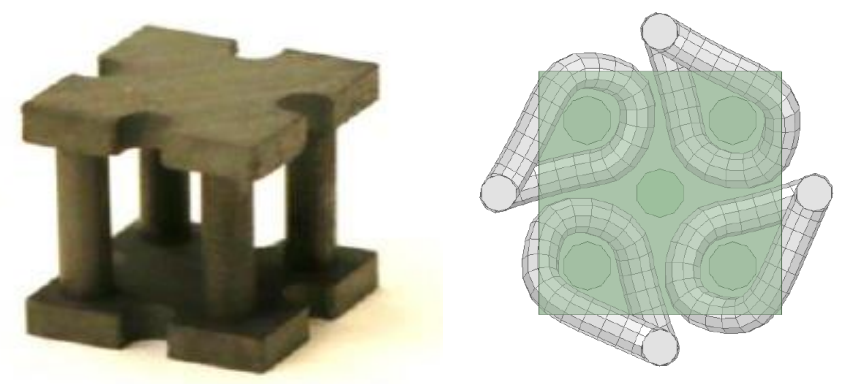

Fig. 1. A multiphase coupled inductor with many coupled windings. There is a center rod providing a returning path for the magnetic flux.

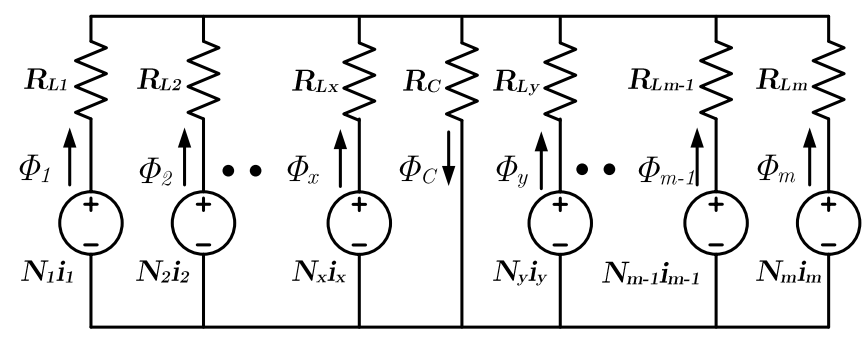

Fig. 2. Reluctance Model of a multiphase coupled inductor with $m$ windings. The $x^{t h}$ winding has $N_{x}$ number of turns. The reluctance of the $x^{t h}$ leg is $\mathcal{R}_{L x}$, and the reluctance of the center rod is $\mathcal{R}_{C}$.

The inductance dual model is a topological dual of a reluctance model of a multileg coupled magnetics. The inductance dual model creates one-to-one linkage between each portion of the magnetic core and the lumped circuit model, leading to simplified design equations for calculating the effective inductance and current ripple in multiphase buck converters. The inductance dual model captures core loss in different part of the core, and allows sophisticated coupling relationship being visualized in SPICE.

The inductance dual model has been occasionally used in previous literature under different names [2]-[4], but to the best of the authors' knowledge, no systematic study has been presented for the inductance dual model. This paper reviews the principles of the multiwinding transformer model and the inductance matrix model, derives the inductance dual model, and presents a systematic approach to compare the interpret the three models.

This paper reviews the three different ways of creating lumped circuit models for coupled magnetics, derives the critical design equations, and compares the advantages and disadvantages of the three models. We also organized the key publications in this field to guide the discussions in this field. 


\section{Reluctance Model}

Fig. 1 shows the picture of a multiphase coupled inductor with $M$ windings each as $N$ number of turns. The physical basis of modeling a multiphase coupled inductor is a reluctance model (Fig. 2). Each winding is coupled to one leg of the magnetic core, and the center rod provides a returning path for the flux. To simplify the analysis and derivation, in the following discussion, we assume that all windings have identical number of turns $N$, and the reluctance of all legs are identically $\mathcal{R}_{L}$. Similar analysis can be extended to cover cases with arbitrary $N . \mathcal{R}_{L}$ and $\mathcal{R}_{C}$ can be freely adjusted to adjust the coupling coefficient between two windings.

By applying Faraday's law and superposition to the reluctance model, a reluctance matrix, $\mathcal{R}_{M \times M}$, can be derived to link the current the $\left(\frac{d i}{d t}\right)$ and the derivative of the magnetic flux going through each winding of the multiple phases:

$\left[\begin{array}{c}N_{1} i_{1} \\ N_{2} i_{2} \\ \vdots \\ N_{M} i_{M}\end{array}\right]=\left[\begin{array}{cccc}\mathcal{R}_{L 1}+\mathcal{R}_{C} & \mathcal{R}_{C} & \cdots & \mathcal{R}_{C} \\ \mathcal{R}_{C} & \mathcal{R}_{L 2}+\mathcal{R}_{C} & \cdots & \mathcal{R}_{C} \\ \vdots & \vdots & \ddots & \vdots \\ \mathcal{R}_{C} & \cdots & \mathcal{R}_{C} & \mathcal{R}_{L M}+\mathcal{R}_{C}\end{array}\right]\left[\begin{array}{c}\Phi_{1} \\ \Phi_{2} \\ \vdots \\ \Phi_{M}\end{array}\right]$

Take the derivative on both sides, and set $N \mathrm{~s}$, and $R_{L} \mathrm{~s}$ as all uniform, $\mathcal{R}_{M \times M}$ links the derivative of current $\left(\frac{d i}{d t}\right)$ and voltage $(v)$ across the $M$ coupled windings:

$N^{2}\left[\begin{array}{c}\frac{d i_{1}}{d t} \\ \frac{d i_{2}}{d t} \\ \vdots \\ \frac{d i_{M}}{d t}\end{array}\right]=\left[\begin{array}{cccc}\mathcal{R}_{L}+\mathcal{R}_{C} & \mathcal{R}_{C} & \cdots & \mathcal{R}_{C} \\ \mathcal{R}_{C} & \mathcal{R}_{L}+\mathcal{R}_{C} & \cdots & \mathcal{R}_{C} \\ \vdots & \vdots & \ddots & \vdots \\ \mathcal{R}_{C} & \cdots & \mathcal{R}_{C} & \mathcal{R}_{L}+\mathcal{R}_{C}\end{array}\right]\left[\begin{array}{c}v_{1} \\ v_{2} \\ \vdots \\ v_{M}\end{array}\right]$

$\mathcal{R}_{M \times M}$, and its inverse matrix, the inductance matrix, $\mathcal{L}_{M \times M}$ serve as the basis of all the derivations and discussions in this paper. All lumped circuit models are developed to represent these two matrices with different focus.

\section{IndUCtANCE MATRIX MODEL}

One way to model the multiphase coupled inductor is to start from the inductance matrix, $\mathcal{L}_{\mathcal{M} \times \mathcal{M}}$, of the coupled inductor, as started from [10] and follow up works:

$$
\left[\begin{array}{c}
v_{1} \\
v_{2} \\
\vdots \\
v_{M}
\end{array}\right]=\left[\begin{array}{cccc}
L_{S} & L_{M} & \cdots & L_{M} \\
L_{M} & L_{S} & \cdots & L_{M} \\
\vdots & \vdots & \ddots & \vdots \\
L_{M} & \cdots & L_{M} & L_{S}
\end{array}\right]\left[\begin{array}{c}
\frac{d i_{1}}{d t} \\
\frac{d i_{2}}{d t} \\
\vdots \\
\frac{d i_{M}}{d t}
\end{array}\right] .
$$

Here $L_{S}$ is the self inductance, and $L_{M}$ is the mutual inductance. Note the inverse of the reluctance matrix is the inductance matrix, $\mathcal{L}_{M \times M}=\mathcal{R}_{M \times M}^{-1}$ in Eq. (2). Applying matrix inverse, $L_{S}$ and $L_{M}$ are functions of $\mathcal{R}_{L}$ and $\mathcal{R}_{C}$ :

$$
\begin{aligned}
& L_{S}=\frac{N^{2}}{\mathcal{R}_{L}+\frac{\mathcal{R}_{L}}{M-1} \| \mathcal{R}_{C}}=\frac{N^{2}\left(\mathcal{R}_{L}+(M-1) \mathcal{R}_{C}\right)}{\mathcal{R}_{L}\left(\mathcal{R}_{L}+M \mathcal{R}_{C}\right)}=\frac{N^{2}}{R_{L}}+L_{M} \\
& L_{M}=\frac{-N^{2}\left(\frac{\mathcal{R}_{L}}{M-2} \| \mathcal{R}_{C}\right)}{\left(\mathcal{R}_{L}+\frac{\mathcal{R}_{L}}{M-1} \| \mathcal{R}_{C}\right)\left(\mathcal{R}_{L}+\frac{\mathcal{R}_{L}}{M-2} \| \mathcal{R}_{C}\right)}=\frac{-N^{2} \mathcal{R}_{C}}{\mathcal{R}_{L}\left(\mathcal{R}_{L}+M \mathcal{R}_{C}\right)}
\end{aligned}
$$

$L_{S}$ and $L_{M}$ can be related to $R_{C}$ and $R_{L}$ :

$$
\mathcal{R}_{L}=\frac{N^{2}}{L_{S}-L_{M}},
$$

$$
\mathcal{R}_{C}=\frac{-N^{2} L_{M}}{\left(L_{S}-L_{M}\right)\left(L_{S}+(M-1) L_{M}\right)} .
$$

$L_{S}$ and $L_{M}$ can also be related to $L_{\mu}$ and $L_{l}$ :

$$
\begin{gathered}
L_{S}=L_{\mu}+L_{l}, \\
L_{M}=-\frac{1}{M-1} L_{\mu} .
\end{gathered}
$$

Note $L_{M}$ is usually negative in a practical design, indicating that the multiple phases are negatively coupled to each other. [10] predicts that the per phase current ripple ratio between noncoupling case and coupling case if the transient equivalent inductance are designed to be the same for a two phase buck converter:

$$
\frac{\Delta i_{c p}}{\Delta i_{\text {noncp }}}=\frac{1+\frac{D}{1-D} \alpha}{1-\alpha}
$$

Here $\alpha=\frac{L_{M}}{L_{S}}=-\frac{\mathcal{R}_{C}}{\mathcal{R}_{L}+\mathcal{R}_{C}}$, and the ripple ratio becomes:

$$
\frac{\Delta I_{c p}}{\Delta I_{\text {noncp }}}=\frac{(1-D) R_{L}+(1-2 D) R_{C}}{(1-D)\left(\mathcal{R}_{L}+2 \mathcal{R}_{C}\right)}
$$

Eq. (20) matches with Eq. (11) for a two winding design. The multiwinding transformer model and the inductance matrix model leads to the same prediction of ripple current reduction.

Four important design parameters for multiphase coupled inductors were defined and derived in [15]:

1) per-phase steady state inductance $\left(L_{p s s}\right)$ : the steady state effective peak -to-peak current ripple per phase;

2) per-phase transient inductance $\left(L_{p t r}\right)$ : the dynamic behavior of the multiphase coupled inductor per phase;

3) overall steady state inductance $\left(L_{o s s}\right)$ : the steady state effective overall output current ripple;

4) overall transient inductance $\left(L_{o t r}\right)$ : the overall dynamic behaviors of the multiphase coupled inductor.

As derived in [15], for a duty ratio of $\frac{k}{M}<D<\frac{k+1}{M}$, the effective per-phase steady state inductance $L_{p s s}$ of the coupled inductor can be calculated using the following equation with elements in the inductance matrix of Eq. (3):

$L_{p s s}=\frac{\left(L_{S}-L_{M}\right)\left(L_{S}+\left(L_{M}-1\right) M\right)}{L_{S}+\left((M-2 k-1)+\frac{k(k-1)}{M D}+\frac{M D(M-2 k-1)+k(k+1)}{M(1-D)} L_{M}\right)}$.

The transient inductance per phase, $L_{p t r}$, is a function of $L_{S}, L_{M}$, and the number of phase $M$, and is independent from the duty ratio $D$ :

$$
L_{p t r}=L_{S}+(M-1) L_{M} .
$$

The overall steady state inductance of the multiphase buck inductor is a function of $k, D, M, L_{M}$, and $L_{S}$ :

$$
L_{o s s}=\frac{(1-D) D M\left(L_{S}+L_{M}(M-1)\right)}{(D M-k)(1+k-D M)}
$$

The overall transient inductance of the multiphase buck inductor is effectively $M$ of the per-phase transient inductance connected in parallel, and is independent from $D$ :

$$
L_{\text {otr }}=\frac{L_{p t r}}{M}=\frac{L_{S}+(M-1) L_{M}}{M} .
$$

With $L_{p s s}, L_{p t r}, L_{o s s}$, and $L_{o t r}$, the overall steady state output current ripple, overall small-signal model, per-phase 
current ripple, and per-phase small-signal model of the multiphase coupled inductor, working in continuous-conductionmode, can be easily estimated by considering the converter as a single phase converter with the same duty ratio $D$ and the effective inductance values.

\section{Multiwinding Transformer Model}

The multiwinding transformer model is first presented in [16] and reference therein to discuss the coupling effects of two-winding coupled inductor. Fig. 3 shows a generalized $n$ winding transformer model. Each winding has a magnetizing inductance $L_{M}^{*}$ and a leakage inductance $L_{L}^{*}$. In a fully symmetric coupled inductor design, $L_{M}^{*} \mathrm{~s}$ and $L_{L}^{*} \mathrm{~s}$ are uniform. In an asymmetric multiphase design, they are different.

The inductance matrix of the multiwinding transformer model is a function of $L_{M}^{*}$ and $L_{L}^{*}$ :

$$
\left[\begin{array}{c}
v_{1} \\
v_{2} \\
\vdots \\
v_{M}
\end{array}\right]=\left[\begin{array}{cccc}
L_{\mu}+L_{l} & -\frac{1}{M-1} L_{\mu} & \cdots & -\frac{1}{M_{1}-1} L_{\mu} \\
-\frac{1}{M-1} L_{\mu} & L_{\mu}+L_{l} & \cdots & -\frac{1}{M-1} L_{\mu} \\
\vdots & \vdots & \ddots & \vdots \\
-\frac{1}{M-1} L_{\mu} & -\frac{1}{M-1} L_{\mu} & \cdots & L_{\mu}+L_{l}
\end{array}\right]\left[\begin{array}{c}
\frac{d i_{1}}{d t} \\
\frac{d i_{2}}{d t} \\
\vdots \\
\frac{d i_{M}}{d t}
\end{array}\right]
$$

$L_{\mu}$ and $L_{l}$ are functions of $R_{L}$ and $R_{C}$ in the reluctance model. Since $R_{C} \gg R_{L}, L_{\mu}$ is dominated by $R_{L}$, and $L_{l}$ is dominated by $R_{C}$.

$$
\begin{gathered}
L_{\mu}=\frac{N^{2}(M-1) \mathcal{R}_{C}}{\mathcal{R}_{L}\left(\mathcal{R}_{L}+M \mathcal{R}_{C}\right)} \approx \frac{(M-1) N^{2}}{M \mathcal{R}_{L}} \\
L_{l}=\frac{N^{2}}{\mathcal{R}_{L}+M \mathcal{R}_{C}} \approx \frac{N^{2}}{M \mathcal{R}_{C}}
\end{gathered}
$$

Define $\rho$ as the ratio between $L_{\mu}$ and $L_{l}$ :

$$
\rho=\frac{L_{\mu}}{L_{l}}=\frac{(M-1) \mathcal{R}_{C}}{\mathcal{R}_{L}}
$$

Assume $D$ is the duty ratio of the multiphase buck converter, $M$ is the number of phases, it is predicted in [18] that if $0<D<\frac{1}{M}$, the ripple ratio between the noncoupling case and coupling case if the same leakage inductance is used for both cases is:

$$
\frac{\Delta i_{c p}}{\Delta i_{\text {noncp }}}=\frac{\frac{M-1+\rho}{M-1+M \rho}-D}{1-D}=\frac{(1-D) \mathcal{R}_{L}+(1-D M) \mathcal{R}_{C}}{(1-D)\left(\mathcal{R}_{L}+M \mathcal{R}_{C}\right)}
$$

This ripple raio is an important figure of merit for designing multiphase coupled magnetics and is widely used in industry. A low ripple ratio indicates higher advantage created by the multiphase coupled inductor.

\section{INDUCTANCE DUAL MODEL}

Fig. 4 shows the inductance dual model, which is a topological dual of the reluctance model in Fig. 2. The reluctance and permanence model can both nicely capture the behavior an asymmetric multiphase coupled inductor. The inductance dual model is a lumped circuit model with inductors and ideal transformers. The cross variable is voltage, and the through variable is current. It can be directly used in SPICE simulations to model coupled inductors in sophisticated circuits. The voltage drop across the inductors and the current flow through the inductors can be converted into the strength of the $B$ field

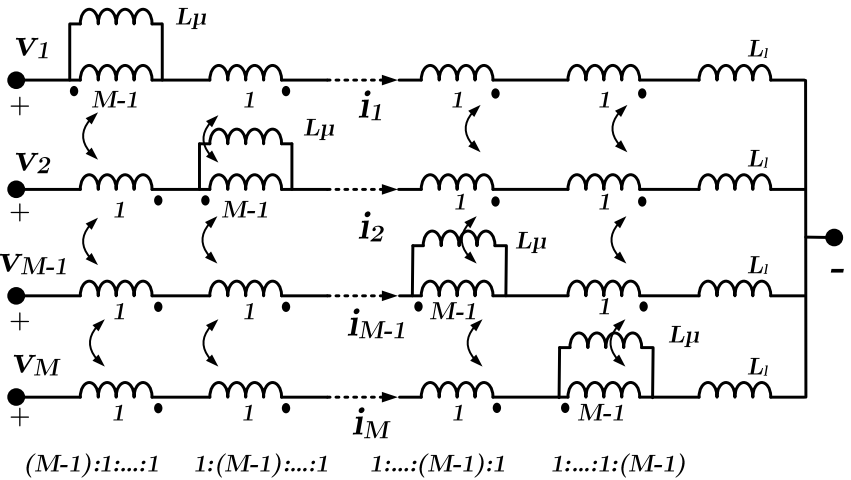

Fig. 3. Multiwinding transformer model of the multiphase coupled inductor. This model reduces to a classic $T$ transformer model for a two winding design.

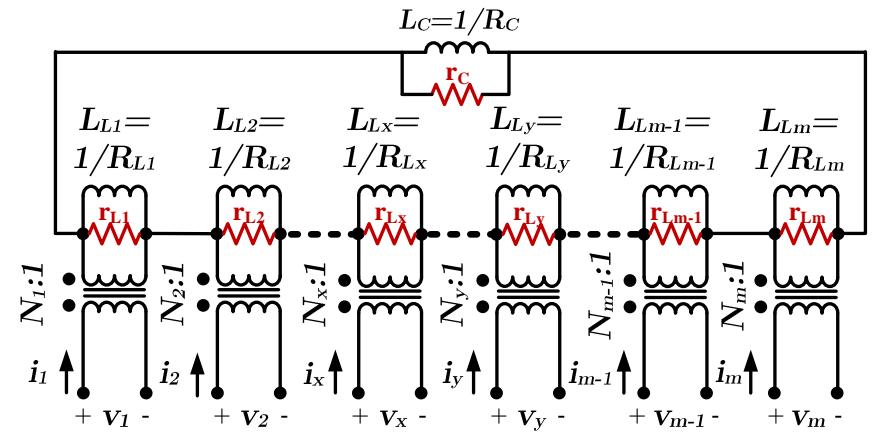

Fig. 4. inductance dual model of the multiphase coupled inductor considering the core loss. $L_{C}$ and $L_{L}$ represent the inductive elements contributed by the center rod and the side legs, respectively. $r_{C}$ and $r_{L}$ capture the loss contributed by the center rod and the side legs, respectively.

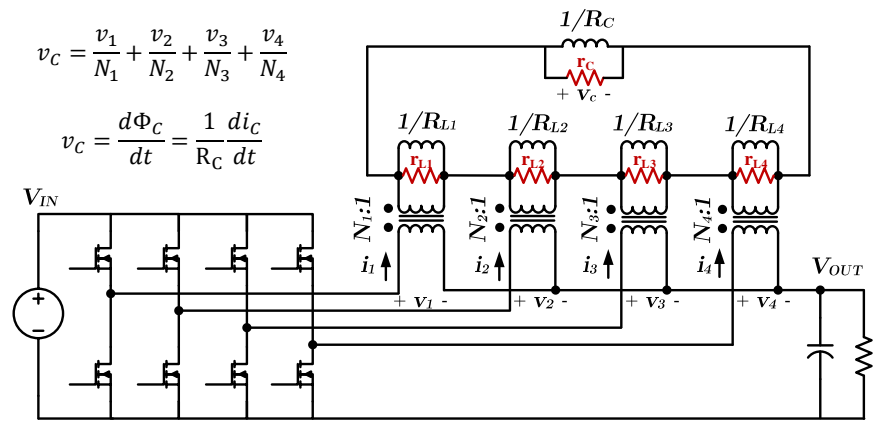

Fig. 5. SPICE simulation of a 4-phase coupled inductor buck converter with a inductance dual model capturing the resistive and inductive behaviors.

and $H$ field, which can be used to model the core loss. In fact, one can add effective resistance $r_{C}$ and $r_{L}$ in parallel with the inductance $L_{C}$ and $L_{L}$, to model the core loss in the legs and rods, respectively. The capability of modeling core loss differently in different core sections is a unique advantage of the inductance dual model. With the inductance dual model in Fig. 4, the derivation of the reluctance matrix, $\mathcal{R}_{M \times M}$ in Eq. 2, becomes very straightforward.

The inductance dual model can be used to derive the steady state output inductance $\left(L_{o s s}\right)$, the transient output inductance $\left(L_{o t r}\right)$, the steady state per-phase inductance $\left(L_{p s s}\right)$, and the transient per-phase inductance $\left(L_{p t r}\right)$, which are identical to 


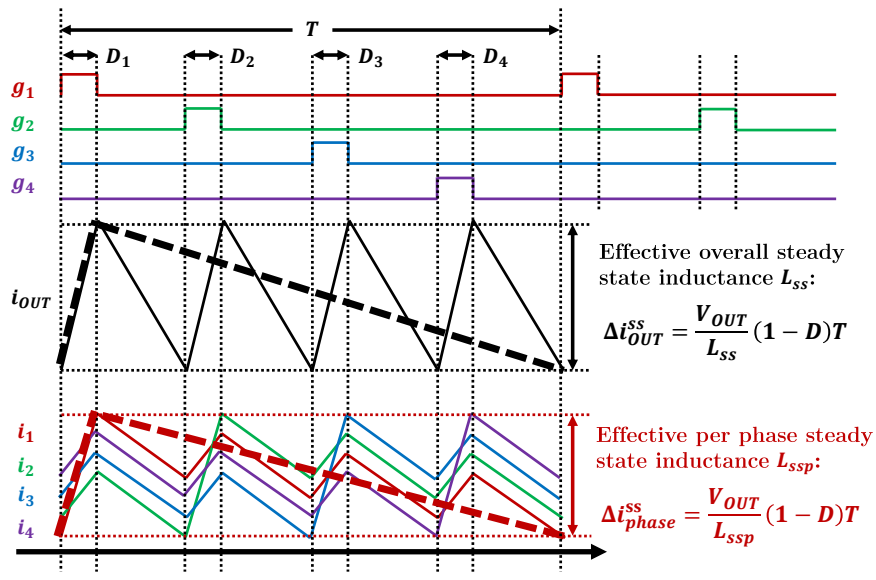

Fig. 6. Effective overall steady state inductance and steady state per-phase inductance of the multiphase coupled inductor.

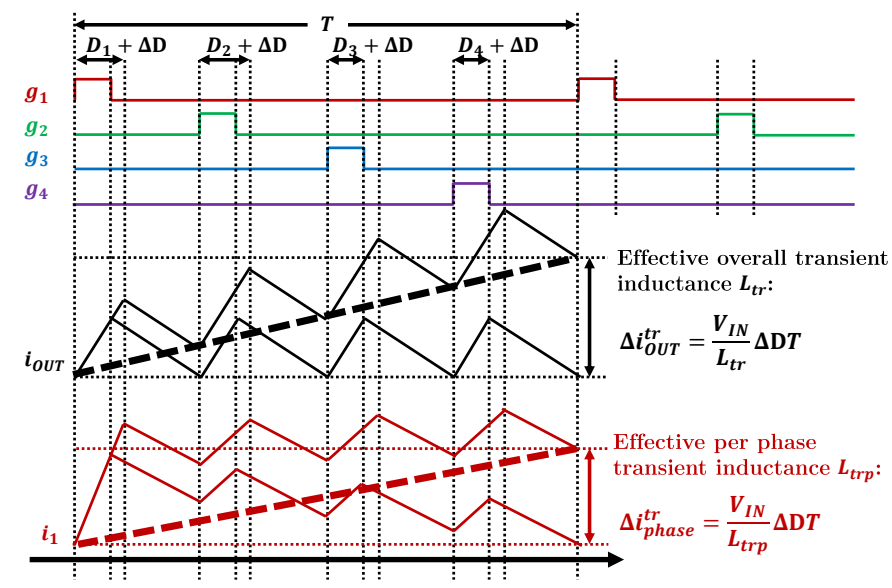

Fig. 7. Effective overall transient inductance and effective per-phase transient inductance of the multiphase coupled inductor.

the inductance matrix model, albeit represented by the reluctance values instead of the self and mutual inductances. Here we present the derivations of the four key design parameters and compare the results.

\section{A. Effective Overall Steady State Inductance}

In a $M$ phase coupled inductor buck converter with $V_{I N}$ as the input voltage and $V_{O U T}$ as the output voltage, for an arbitrary duty ratio selection $\frac{k}{M}<D<\frac{k+1}{M}$, the derivative of the overall output current is the summation of the derivative of the current of all phases:

$$
\frac{d i_{O U T}}{d t}=\frac{d i_{1}}{d t}+\frac{d i_{2}}{d t}+\ldots+\frac{d i_{M}}{d t} .
$$

- During $\frac{k}{M} T<t<D T$, the overall current ramps up. There is always $k+1$ inductors taking $V_{I N}-V_{O U T}$, and $M-k-1$ inductors taking $-V_{O U T}$ for an $\mathrm{ON}$ period $\left(D-\frac{k}{M}\right) T$. The $k+1^{\text {th }}$ inductor is taking $V_{I N}-V_{O U T}$.

- During $D T<t<\frac{k+1}{M} T$, the overall current ramps down. There is always $i$ inductors taking $V_{I N}-V_{O U T}$, and $M-$ $k$ inductors taking $-V_{O U T}$ for an OFF period $\left(\frac{k+1}{M}-\right.$ $D) T$. The $k+1^{t h}$ inductor is taking $-V_{O U T}$.
Substituting Eq. (21) into Eq. (2), adding all elements in the matrix together, the current ripple during the ramp up and ramp down period are:

$$
\begin{aligned}
\Delta i_{\text {OUT }}^{u p} & =\frac{\left(\mathcal{R}_{L}+M \mathcal{R}_{C}\right)(k+1-D M)(D M-k)}{D M N^{2}} V_{O U T} T \\
\Delta i_{O U T}^{\text {down }} & =-\frac{\left(\mathcal{R}_{L}+M \mathcal{R}_{C}\right)(k+1-D M)(D M-k)}{D M N^{2}} V_{O U T} T
\end{aligned}
$$

Note the steady state output current ripple, $\Delta i_{O U T}^{s s}=$ $\Delta i_{\text {OUT }}^{\text {up }}=-\Delta i_{\text {OUT }}^{\text {down }}=\frac{V_{O U T}}{L_{s s}}(1-D) T$. The effective multiphase steady state inductance $L_{o s s}$ of a $M$ phase coupled buck converter with $\frac{k}{M}<D<\frac{k+1}{M}$ is:

$$
L_{o s s}=\frac{(1-D) D M N^{2}}{\left(\mathcal{R}_{L}+M \mathcal{R}_{C}\right)(k+1-D M)(D M-k)}
$$

For an $M$-phase coupled inductor buck converter operating in CCM with duty ratio $D$, switching period $T$, and output voltage $V_{O U T}$, the peak-to-peak current ripple of the overall output current is:

$$
\Delta i_{O U T}=\frac{V_{O U T}(1-D) T}{L_{o s s}}
$$

If $\mathcal{R}_{C}=0$, all phases are not coupled. $L_{o s s}$ is the effective steady state inductance if $M$ inductors with individual inductance of $\frac{N^{2}}{R_{L}}$ operated in interleaving.

$$
L_{\text {oss-noncp }}=\frac{N^{2}}{\mathcal{R}_{L}} \frac{(1-D) D M}{(k+1-D M)(D M-k)} .
$$

\section{B. Effective Per-Phase Steady State Inductance}

If $\frac{k}{M}<D<\frac{k+1}{M}$, during the $0<t<D T$ period, for each phase, there are $k+1$ numbers of $\left(D-\frac{k}{M}\right) T$ sub-periods, in which $k+1$ phases are driven by $V_{I N}-V_{O U T}$, and $M-k-1$ phases are driven by $-V_{O U T}$. The per-phase current ramps up during this period. The current ripple in each winding during the $k+1$ numbers of $\left(D-\frac{k}{M}\right) T$ sub-periods is:

$$
\begin{aligned}
\Delta i_{\text {phase }}^{\text {up }}= & \left(\left(\mathcal{R}_{L}+\mathcal{R}_{C}+k \mathcal{R}_{C}\right) \frac{1-D}{D}-(M-k-1) \mathcal{R}_{C}\right) \\
& \times\left(D-\frac{k}{M}\right) \frac{(k+1) T V_{\text {OUT }}}{N^{2}} .
\end{aligned}
$$

During the $k$ numbers of $\left(\frac{k+1}{M}-D\right) T$ subperiods, $k$ phases are driven by $V_{I N}-V_{O U T}$, and $M-k$ phases are driven by $-V_{O U T}$. The per-phase current ramps down during this period. The current ripple in each phase during the $k$ numbers of $\left(\frac{k+1}{M}-D\right) T$ subperiod is:

$$
\begin{aligned}
\Delta i_{\text {phase }}^{\text {down }}= & \left(\left(\mathcal{R}_{L}+\mathcal{R}_{C}+(k-1) \mathcal{R}_{C}\right) \frac{1-D}{D}-(M-k) \mathcal{R}_{C}\right) \\
& \times\left(\frac{k+1}{M}-D\right) \frac{k T V_{\text {OUT }}}{N^{2}} .
\end{aligned}
$$

The current ripple in each phase is the summation of all the ramp up and ramp down sub-periods:

$$
\begin{aligned}
& \Delta i_{\text {phase }}^{\text {ss }}=\Delta i_{\text {phase }}^{\text {up }}+\Delta i_{\text {phase }}^{\text {down }}= \\
& \frac{T V_{\text {OUT }}}{N^{2}}\left(-\frac{k^{2} \mathcal{R}_{C}}{D M}-\frac{k \mathcal{R}_{C}}{D M}-D \mathcal{R}_{L}-D M \mathcal{R}_{C}+2 k \mathcal{R}_{C}+\mathcal{R}_{L}+\mathcal{R}_{C}\right) .
\end{aligned}
$$


Define $L_{p s s}$ as the effective inductance of each winding in steady state $\Delta i_{\text {phase }}^{s s}=\frac{V_{O U T}}{L_{p s s}}(1-D) T$ :

$$
L_{p s s}=\frac{N^{2}(1-D)}{-\frac{k^{2} \mathcal{R}_{C}}{D M}-\frac{k \mathcal{R}_{C}}{D M}+2 k \mathcal{R}_{C}-D M \mathcal{R}_{C}+\mathcal{R}_{C}-D \mathcal{R}_{L}+\mathcal{R}_{L}} .
$$

For an $M$-phase coupled inductor buck converter operating in CCM with duty ratio $D$, switching period $T$, and output voltage $V_{O U T}$, the peak-to-peak current ripple of the per-phase winding current is:

$$
\Delta i_{\text {phase }}=\frac{V_{\text {OUT }}(1-D) T}{L_{p s s}} .
$$

\section{Effective Overall Transient Inductance}

The transient inductance determines the response of $i_{\text {OUT }}$ to a small purtubation in $D$. For a buck converter with a discrete inductor, the ramp up slope of the inductor current is $\frac{d i_{O U T}}{d t} u p=\frac{V_{I N}-V_{\text {OUT }}}{L}$, the ramp down slope of the inductor current is $\frac{d i_{\text {OUT }}}{d t}$ down $=\frac{-V_{\text {OUT }}}{L}$. If there is a small pertubation $\Delta D, i_{O U T}$ will ramp up $\frac{V_{I N}-V_{O U T}}{L} \Delta D$ more, and will ramp down $\frac{V_{O U T}}{L} \Delta D$ less. As a result, the perturbation in the inductor current is $\frac{V_{I N}}{L} \Delta D T$. In other words, the transient inductance of a single-phase buck converter with $L$ is still $L$. The transient inductance determines the system dynamic performance and can be used in small signal models. One typical design goal for multiphase coupled inductor is to maximize the steady state inductance and minimize the transient inductance. For a $M$ phase coupled inductor buck converter, if $\frac{k}{M}<D<\frac{k+1}{M}$, the ramp up and ramp down rate of the output current is:

$$
\begin{aligned}
& \frac{d i_{\text {OUT }}^{t r-u p}}{d t}=\frac{\left(\mathcal{R}_{L}+M \mathcal{R}_{C}\right)(k+1-D M)}{D N^{2}} V_{O U T}, \\
& \frac{d i_{\text {OUT }}^{\text {tr-down }}}{d t}=\frac{\left(\mathcal{R}_{L}+M \mathcal{R}_{C}\right)(k-D M)}{D N^{2}} V_{O U T} .
\end{aligned}
$$

Note there are $M$ ripple cycles within a switching period $T$. A perturbation $\Delta D$ results in a perturbation of $\left(\frac{d i_{O U T}^{t_{p}}}{d t}-\right.$ $\left.\frac{d i_{O U T}^{\text {down }}}{d t}\right) D M T$, which equals $\frac{\left(\mathcal{R}_{L}+M \mathcal{R}_{C}\right)}{N^{2}} V_{I N} \Delta D M T$ in the overall output current. Since

$$
\Delta i_{\text {OUT }}^{t r}=\frac{V_{I N}}{L_{t r}} \Delta D T=\frac{\left(\mathcal{R}_{L}+M \mathcal{R}_{C}\right)}{N^{2}} V_{I N} \Delta D M T,
$$

the effective overall transient inductance of a $M$ phase coupled inductor, regardless of the duty ratio $D$, is:

$$
L_{t r}=\frac{N^{2}}{M\left(\mathcal{R}_{L}+M \mathcal{R}_{C}\right)}
$$

$L_{t r}$ can be used deriving the small signal model of multiphase coupled inductor buck converters.

\section{Effective Per-Phase Transient Inductance}

Since the current equally distribute among the $M$ parallel windings, with a purtubation of $\Delta D$ in the duty ratio, the perturbation in the current in each winding is $\frac{1}{M}$ of the overall current perturbation. As a result:

$$
\frac{V_{I N}}{L_{t r w}} \Delta D T=M \frac{\left(\mathcal{R}_{L}+M \mathcal{R}_{C}\right)}{N^{2}} V_{I N} \Delta D T \times \frac{1}{M} .
$$

The effective per-phase transient inductance of a $M$ phase coupled inductor, regardless of the duty ratio $D$, is:

$$
L_{p t r}=\frac{N^{2}}{\mathcal{R}_{L}+M \mathcal{R}_{C}}
$$

\section{E. Summary of the Design Equations}

We use the inductance dual model to derive the ratio between the per phase current ripple of a noncoupled design case and a coupled case if the transient equivalent inductance are the same for a two phase buck converter. Assuming the transient equivalent inductances for both cases are $L_{p t r}=\frac{N^{2}}{\mathcal{R}_{L}+M \mathcal{R}_{C}}$. The steady state current ripple per phase is Eq. (29):

$$
\begin{aligned}
& \Delta i_{c p}= \\
& \frac{V_{O U T} T}{N^{2}}\left(-\frac{k^{2} \mathcal{R}_{C}}{D M}-\frac{k \mathcal{R}_{C}}{D M}+2 k \mathcal{R}_{C}-D \mathcal{R}_{L}-D M \mathcal{R}_{C}+\mathcal{R}_{L}+\mathcal{R}_{C}\right) .
\end{aligned}
$$

If all the inductors are not coupled, and each of them has an inductance of $L_{p t r}=\frac{N^{2}}{\mathcal{R}_{L}+M \mathcal{R}_{C}}$, the steady state current ripple per phase is:

$$
\Delta i_{n o n c p}=\frac{V_{O U T} T}{L_{p t r}}(1-D)=\frac{V_{O U T} T}{N^{2}}\left(\mathcal{R}_{L}+M \mathcal{R}_{C}\right)(1-D) .
$$

If $0<D<\frac{1}{M}, k=0$, the per-phase current ripple ratio between the noncoupling case and coupling case if the same leakage inductance is used for both cases is:

$$
\frac{\Delta i_{c p}}{\Delta i_{\text {noncp }}}=\frac{(1-D) \mathcal{R}_{L}+(1-D M) \mathcal{R}_{C}}{(1-D)\left(\mathcal{R}_{L}+M \mathcal{R}_{C}\right)} .
$$

which is identical to Eq. (20), originally derived in [18].

If $M=2$ and $k=0$, the ripple ratio between the noncoupled case and coupled cases is:

$$
\frac{\Delta i_{c p}}{\Delta i_{\text {noncp }}}=\frac{(1-D) \mathcal{R}_{L}+(1-2 D) \mathcal{R}_{C}}{(1-D)\left(\mathcal{R}_{L}+2 \mathcal{R}_{C}\right)},
$$

which is identical to Eq. (11), originally derived in [10].

As a result, the equations derived by the permenance model match exactly with the existing design equations and offer new insights. The inductance dual model describes the same KVL and KCL equations with a lumped circuit model which has significantly reduced circuit complexity and clear physical meaning, can reveal the flux density in different section of the core, while retaining the same accuracy and effectiveness as all existing methods. Table I summarizes and compares all design equations derived based on the three models.

\section{Design Figure of Merits}

As stated in [15], $L_{o s s}, L_{o t r}, L_{p s s}, L_{p t r}$ can be used to define a few figure of merits (FOM) as design guidelines for multiphase coupled inductors. $L_{o t r}$ and $L_{o s s}$ determines the multi-phase performance. For a given $L_{o t r}$, a higher $L_{o s s}$ indicates smaller output current ripple, smaller output capacitance, and faster transient. $L_{p t r}$ and $L_{p s s}$ determines the per-phase performance. For a given $L_{p t r}$, a higher $L_{p s s}$ indicates smaller conduction loss, core loss, and higher efficiency. We define a 

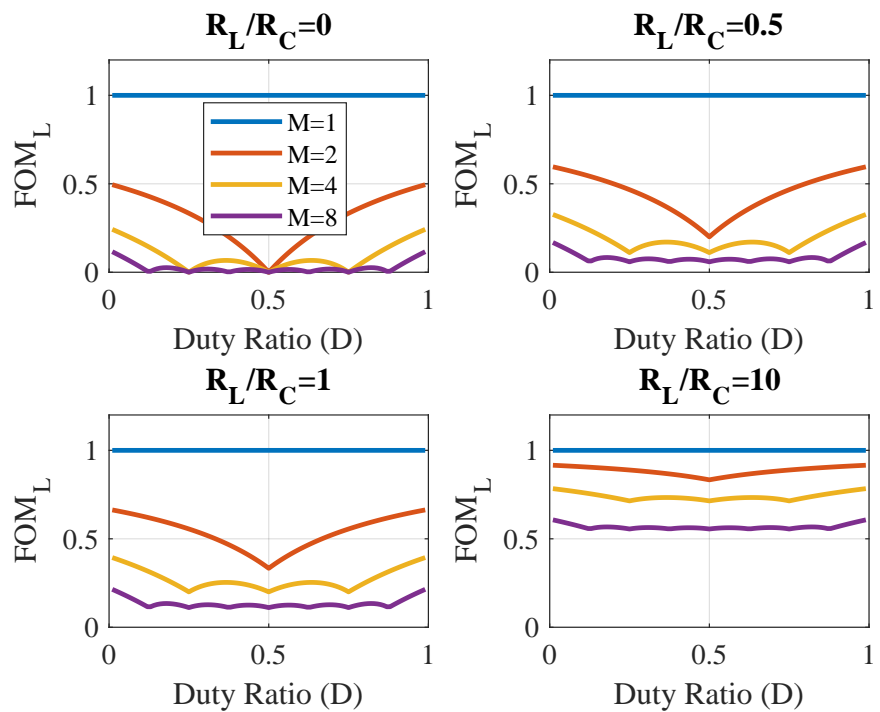

Fig. 8. Inductor size figure of merits $\left(F O M_{L}\right)$ of the multiphase coupled inductor as functions of the number of phases $(M)$, duty ratio $(D)$, and reluctance ratio $\left(\beta=\mathcal{R}_{L} / \mathcal{R}_{C}\right)$. A high reluctance ratio indicates weak coupling, and a low reluctance ratio indicates strong coupling.

FOM as the following to estimate the current ripple reduction per-phase for a given transient inductance:

$$
\begin{aligned}
& \text { FOM }_{L}=\frac{L_{p t r}}{L_{p s s}}=\frac{\Delta i_{c p}}{\Delta i_{\text {noncp }}} \\
& =\frac{-\frac{k^{2} \mathcal{R}_{C}}{D M}-\frac{k \mathcal{R}_{C}}{D M}+2 k \mathcal{R}_{C}-D M \mathcal{R}_{C}+\mathcal{R}_{C}-D \mathcal{R}_{L}+\mathcal{R}_{L}}{(1-D)\left(\mathcal{R}_{L}+M \mathcal{R}_{C}\right)} .
\end{aligned}
$$

If $\beta=\frac{\mathcal{R}_{L}}{\mathcal{R}_{C}}$, FOM becomes:

$$
F O M_{L}=\frac{-\frac{k^{2}}{D M}-\frac{k}{D M}+2 k-D M+1+\beta-D \beta}{(1-D)(\beta+M)} .
$$

Fig. 9 plots the $F O M_{L}$ with a range of $D, M$, and $\beta$. The $F O M_{L}$ is always higher than zero and smaller than one. A smaller $F O M_{L}$ indicates smaller effective transient inductance and faster system transient response. If $\beta \rightarrow 0, \mathcal{R}_{L} \ll \mathcal{R}_{C}$, the inductors are strongly uncoupled, the benefits of coupling show up on each single phase. If $\beta \rightarrow+\infty, \mathcal{R}_{L} \gg \mathcal{R}_{C}$, the inductors are weakly coupled, and $F O M \rightarrow 1$, indicating that there is almost no benefits from coupling.

We can also define the current ripple ratio between a multiphase coupled inductor and a single phase inductor as the figure of merit. When $D=0.5, M=1, k=0$, the effective inductance is $L_{p s s}=\frac{N^{2}}{\mathcal{R}_{C}+\mathcal{R}_{L}}$. The current ripple on the inductor reaches the maximum:

$$
\Delta i_{O U T}=\frac{V_{I N} T\left(\mathcal{R}_{C}+\mathcal{R}_{L}\right)}{4 N^{2}} .
$$

The per phase current ripple on the inductor is:

$$
\begin{aligned}
& \Delta i_{\text {phase }}= \\
& \frac{V_{I N} D T}{N^{2}}\left(-\frac{k^{2} \mathcal{R}_{C}}{D M}-\frac{k \mathcal{R}_{C}}{D M}+2 k \mathcal{R}_{C}-D M \mathcal{R}_{C}+\mathcal{R}_{C}-D \mathcal{R}_{L}+\mathcal{R}_{L}\right) .
\end{aligned}
$$

The normalized current ripple is:

$$
F O M_{I}=\frac{4 D\left(-\frac{k^{2}}{D M}-\frac{k}{D M}+2 k-D M+1-D \beta+\beta\right)}{1+\beta}
$$
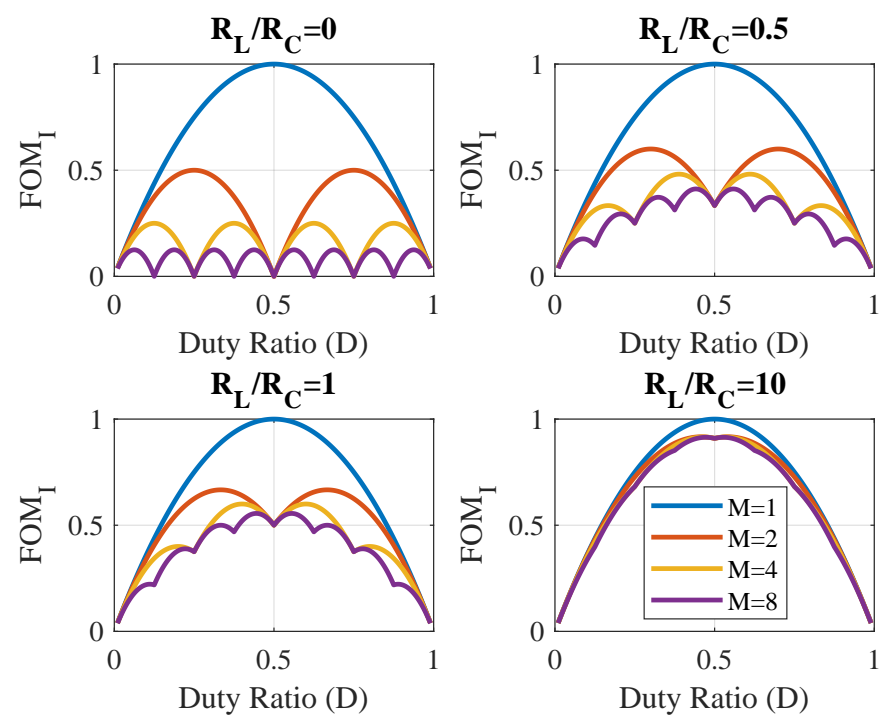

Fig. 9. Current ripple figure of merits $\left(F O M_{I}\right)$ of the multiphase coupled inductor as functions of the number of phases $(M)$, duty ratio $(D)$, and reluctance ratio $\left(\beta=\mathcal{R}_{L} / \mathcal{R}_{C}\right)$. A high reluctance ratio indicates weak coupling, and a low reluctance ratio indicates strong coupling.

Fig. 9 plots the $F O M_{I}$ across a range of $D, M$, and $\beta$. The $F O M_{I}$ is always larger than zero and smaller than one. A smaller $F O M_{I}$ indicates smaller steady state current ripple. If $k \rightarrow 0, \mathcal{R}_{L} \ll \mathcal{R}_{C}$, inductors are strongly coupled, the benefits of coupling increases. If $k \rightarrow+\infty, \mathcal{R}_{L} \gg \mathcal{R}_{C}$, inductors are weakly coupled, and $F O M_{I} \rightarrow 1$, indicating no benefits. $F O M_{I}$ becomes independent from the number of phases.

\section{DESIGN VERIFICATIONS}

We use a four phase coupled inductor buck converter with $\mathcal{R}_{L}=2,000,000$ Ampere-turn/Weber, and $\mathcal{R}_{C}=2,000,000$ Ampere-turn/Weber to verify the effectiveness of the three methods. The inductance values in the inductance dual model for this circuit are $L_{L}=500 \mathrm{nH}$, and $L_{C}=500 \mathrm{nH}$. $L_{l}=100 \mathrm{nH}$ and $L_{\mu}=300 \mathrm{nH}$ for the multiwinding transformer model. Assume $M=4, D=40 \%$, and $k=1$, according to Table I, the effective overall steady state inductance of this coupled inductor $L_{o s s}$ is $400 \mathrm{nH}$. The overall transient inductance of this coupled inductor $L_{o t r}$ is $25 \mathrm{nH}$. The effective per-phase steady state inductance of this coupled inductor $L_{p s s}$ is $400 \mathrm{nH}$. The effective transient inductance per phase of this coupled inductor $L_{p t r}$ is $100 \mathrm{nH}$.

For an input voltage of $8 \mathrm{~V}$ and a duty ratio of $40 \%$, with a switching frequecy of $1 \mathrm{MHz}$, the steady state output current ripple is $4.8 \mathrm{~A}$, the steady state per phase current ripple is $4.8 \mathrm{~A}$. In comparison, if we need to achieve the same transient response, the uncoupled inductance per phase should be $100 \mathrm{nH}$. The current ripple per phase is $19.2 \mathrm{~A}$. The effective steady state inductance with this four phase interleaved non coupled inductor is $400 \mathrm{nH}$, and the steady state output current ripple is also $4.8 \mathrm{~A}$. By doing interleaving and multiphase coupling, while maintain the same transient inductance, and the same output current ripple, the steady state per chase current ripple is reduced from $19.2 \mathrm{~A}$ to $4.8 \mathrm{~A}$. 
TABLE I

UNIFIEd MODELS FOR MULTIPHASE COUPLED INDUCTORS

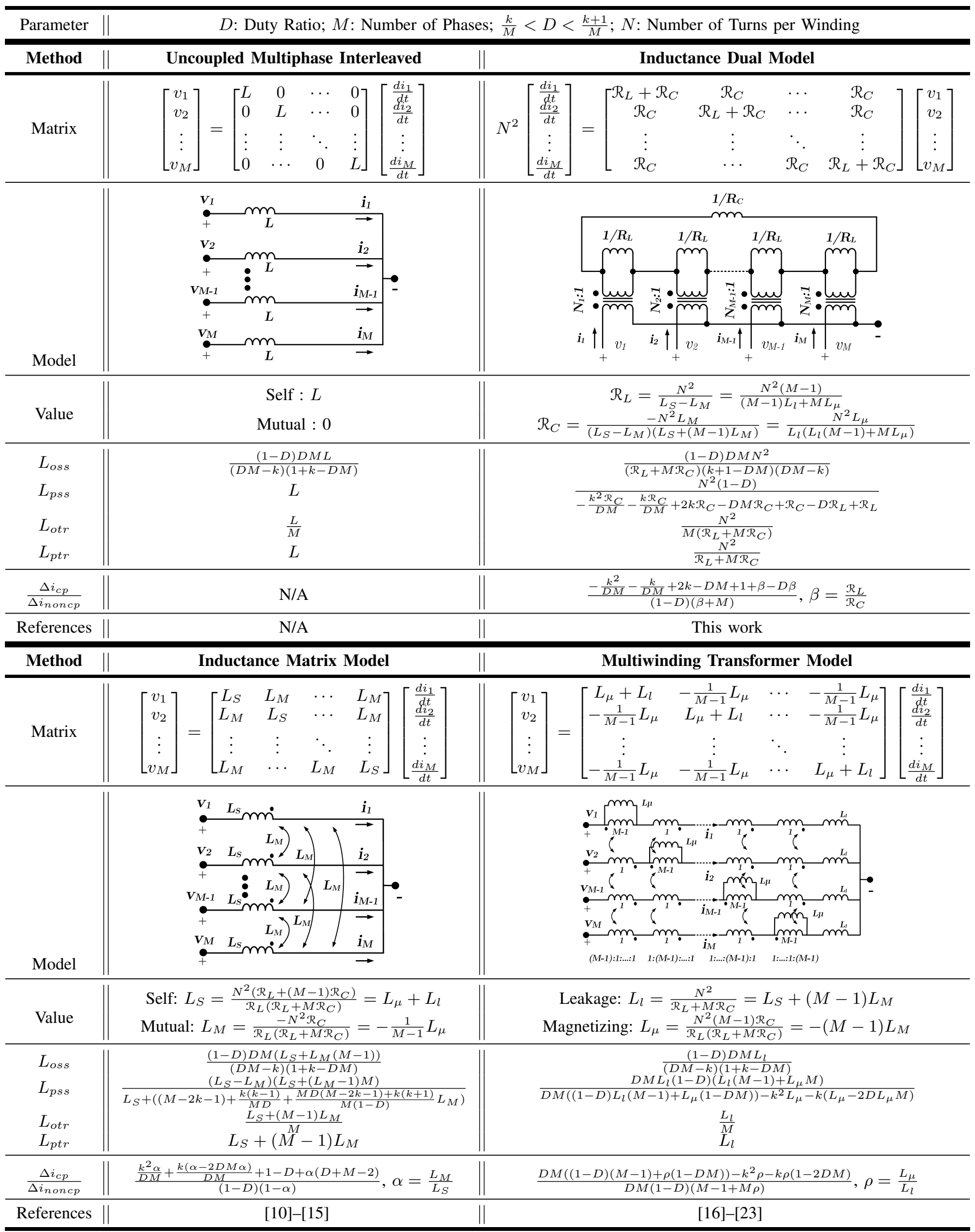




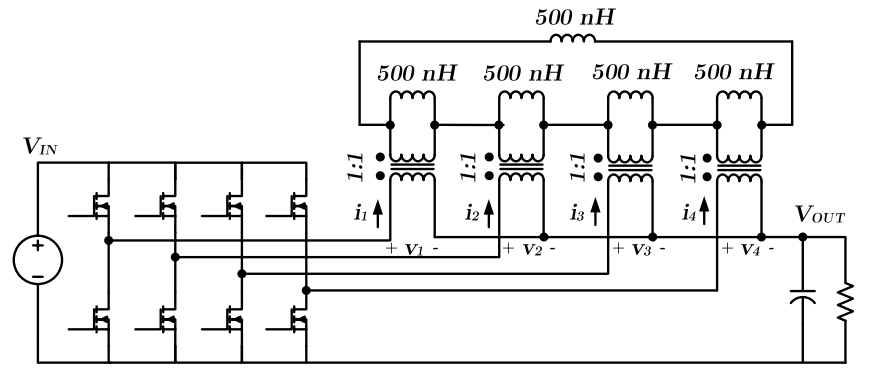

Fig. 10. An example four phase coupled inductor buck converter with $R_{C}=R_{L}=2,000,000$ Ampere-turn/Weber. Each phase has a single turn.

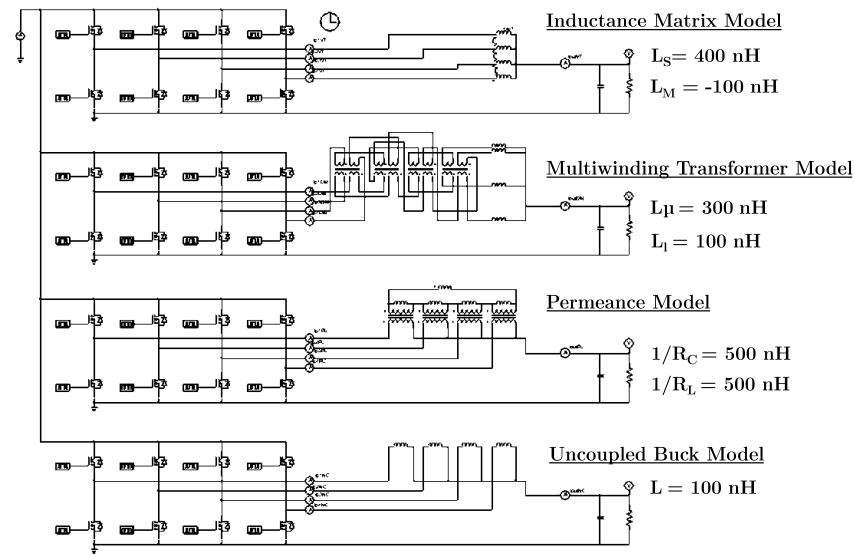

Fig. 11. SPICE simulation platform of a four phase buck converter with a four-winding coupled inductor. The self inductance $\left(L_{S}\right)$ is $400 \mathrm{nH}$, and the mutual inductance $\left(L_{M}\right)$ is $-100 \mathrm{nH}$. The magnetizing inductance $L_{\mu}$ is $300 \mathrm{nH}$, and the leakage inductance $L_{l}$ is $100 \mathrm{nH}$. The central and side inductances $\left(L_{C}\right.$ and $\left.L_{L}\right)$ are both $500 \mathrm{nH}$. The four circuits have identical transient inductance but different steady state inductances.

Fig. 11 compares four SPICE models for the multiphase coupled inductor buck converter. The first model was developed based on the inductance matrix with self and mutual inductance. The second circuit model was developed based on the multiwinding transformer model with magnetizing and leakage inductance. The third circuit is the inductance dual model. The fourth circuit is an uncoupled multiphase buck inductor. The first three circuit model the same coupled inductor structure and have identical modeling results. The fourth circuit model has the same transient behavior as the top three circuit model (as shown in Fig. 11), but suffers larger per phase current ripple (thus higher loss and lower efficiency).

Fig. 12 show the simulated transient and steady state waveforms. As predicted, the inductance matrix model, the multiwinding transformer model, and the inductance dual model predicts exactly the same steady state and transient performance of the four-phase coupled inductor buck converter. The waveform overlaps perfectly with each other (both in transient and in steady state). Also, as predicted, by doing multiphase coupling and interleaving, the transient performance of the coupled inductor buck and a standard four phase interleaved buck are maintained the same, but the per phase current ripple is reduced from 19.2 A to $4.8 \mathrm{~A}$. The simulated results match perfectly with the theoretical calculation. The transients of the output voltage waveforms are also exactly the same.
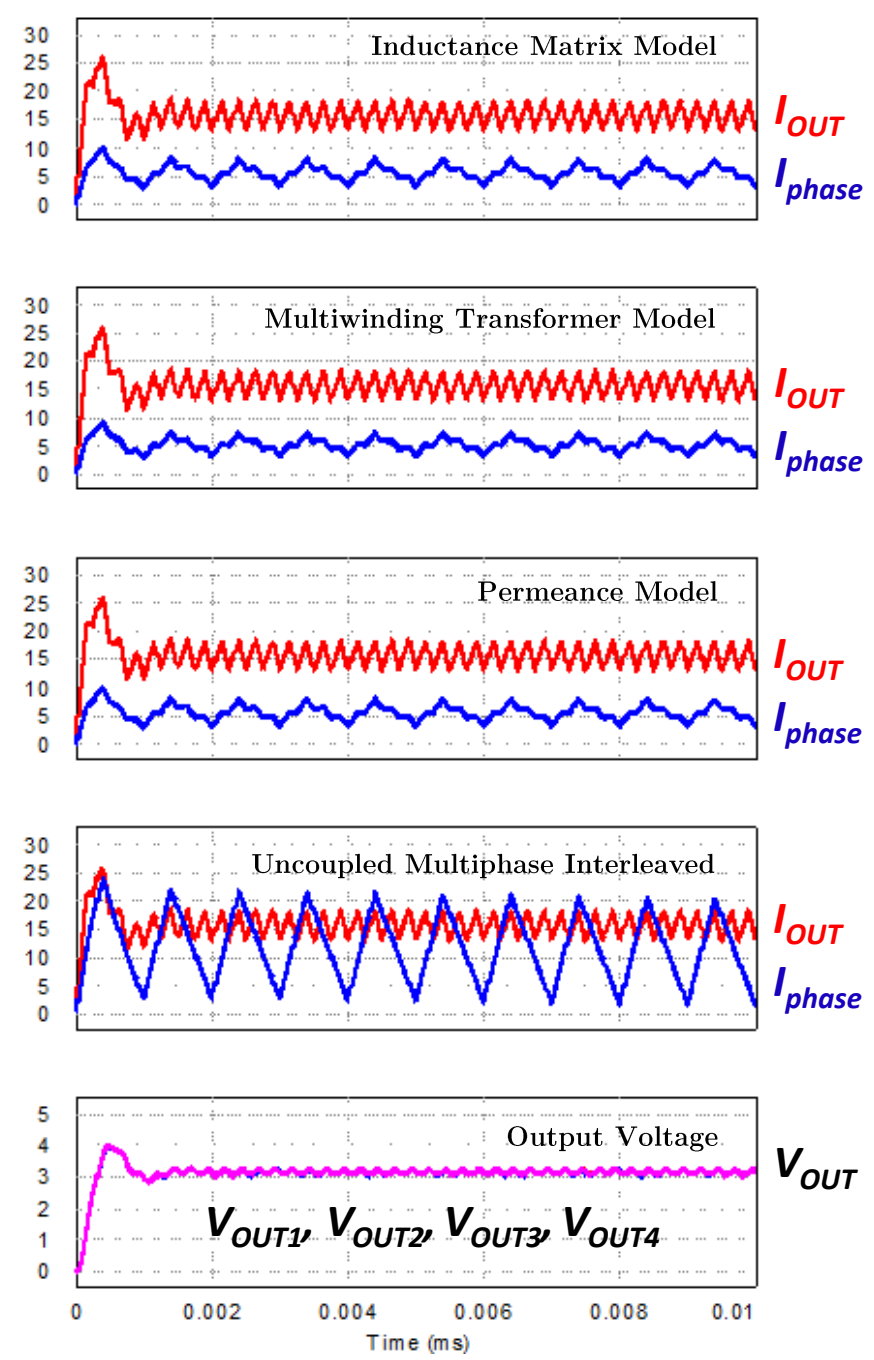

Fig. 12. Simulated waveforms of the four circuit models. The inductance matrix, multiwinding transformer and inductance dual models predict the same circuit behaviors. The SPICE simulated ripples match perfectly with calculated ripples. The steady state total output current ripples are $4.8 \mathrm{~A}$. The steady state per phase current ripple of the coupled inductor buck is $4.8 \mathrm{~A}$, while the per phase current ripple of a noncoupled inductor is $19.2 \mathrm{~A}$. The per phase current ripple is significantly reduced with the same transient inductance.

\section{CONCLUSIONS}

This letter unifies the models for coupled inductors in multiphase buck converters. The inductance dual model is a topological duality of a traditional transformer model, and is derived based on the reluctance matrix instead of the inductance matrix. The inductance dual model creates intuitive linkage between the geometry information of the coupled inductor structure and the lumped circuit model, and significantly simplifies the equations for calculating the effective inductance and current ripple in multiphase buck converters. It allows sophisticated electromagnetic coupling being visualized in SPICE simulations. The inductance dual model is rigorously verified through analytical derivations and SPICE simulations. The equations derived based on the inductance dual model are identical to the equations derived with the ideal transformer model, and can offer additional physical insights and design guidelines for multiphase buck converters. 


\section{ACKNOWLEDGEMENTS}

This work was supported by the National Science Foundation under Award \#1847365.

\section{REFERENCES}

[1] D. C. Prince, "Rectifier voltage control," Journal of the A.I.E.E., vol. 45, no. 7, pp. 630-636, July 1926.

[2] I. G. Park and S. I. Kim, "Modeling and analysis of multiinterphase transformers for connecting power converters in parallel," IEEE Power Electronics Specialists Conference, Saint Louis, MO, USA, 1997, pp. 1164-1170, vol.2.

[3] S. El-Hamamsy and E. I. Chang, "Magnetics modeling for computer-aided design of power electronics circuits," IEEE Power Electronics Specialists Conference, Milwaukee, WI, USA, 1989, pp. 635-645 vol.2.

[4] G. W. Ludwig and S. El-Hamamsy, "Coupled inductance and reluctance models of magnetic components," IEEE Transactions on Power Electronics, vol. 6, no. 2, pp. 240-250, April 1991.

[5] D. C. Hamill, "Lumped equivalent circuits of magnetic components: the gyrator-capacitor approach," IEEE Transactions on Power Electronics, vol. 8, no. 2, pp. 97-103, April 1993.

[6] D. C. Hamill, "Gyrator-Capacitor Modeling: A Better Way of Understanding Magnetic Components," IEEE Applied Power Electronics Conference, vol. 1, 13-17 Feb 1994, pp.326- 32, Orlando, FL.

[7] B. Cougo, "Design and Optimization of InterCell Transformers forParallel MultiCell Converters," Ph.D. Thesis, University de Toulouse, 2011.

[8] F. M. Lambert, J. Mahseredjian, M. Martinez-Duro and F. Sirois, "Magnetic Circuits Within Electric Circuits: Critical Review of Existing Methods and New Mutator Implementations," IEEE Transactions on Power Delivery, vol. 30, no. 6, pp. 2427-2434, Dec. 2015.

[9] X. Zhou, P.-L. Wong, P. Xu, F. C. Lee, and A. Q. Huang, "Investigation of candidate VRM topologies for future microproceossrs," IEEE Trans. Power Electron., vol. 15, pp. 1172-1182, Nov. 2000.

[10] P.-L. Wong, P. Xu, P. Yang, and F.C. Lee, "Performance Improvements of Interleaving VRMs with Coupling Inductors," IEEE Trans. on Power Electronics, vol. 16, no. 4, pp. 499-507, 2001.

[11] P.-L. Wong, "Performance improment of multi-channel interleaving voltage regulator modules with integrated coupling inductors", Ph.D dissertation, Virginia Tech, Blacksburg, VA, Mar., 2001.

[12] P.-L. Wong, F. C. Lee, Peng Xu and Kaiwei Yao, "Critical inductance in voltage regulator modules," IEEE Transactions on Power Electronics, vol. 17, no. 4, pp. 485-492, July 2002.

[13] P. Xu, J. Wei, K. Yao, Y. Meng, F.C. Lee, "Investigation of Candidate Topologies for 12 V VRM," IEEE Applied Power Electronics Conference and Exposition, APEC 2002, pp. 686-692 vol.2.

[14] Z. Lu, W. Chen, "Multi-phase inductor coupling scheme with balancing winding in VRM applications", in Proc. IEEE APEC, 2007, pp.731-735

[15] Y. Dong, "Investigation of Multiphase Coupled-Inductor Buck Converters in Point-of-Load Applications", PhD Thesis, Virginia Tech, 2009.

[16] J. Li, T. Abdallah, and C.R. Sullivan, "Improved calculation of core loss with non-sinusoidal waveforms", Proc. IEEE IAS 2001, pp.2203-2210.

[17] J. Li, C. R. Sullivan, and A. Schultz, "Coupled Inductor Design Optimization for Fast-response Low-Voltage DC-DC Converters," IEEE Applied Power Electronics Conference and Exposition, APEC 2002, pp. 817-823 vol.2.

[18] J. Li, A. Stratakos, C. R. Sullivan, and A. Schultz, "Using Coupled Inductors to Enhance Transient Performance of Multi-Phase Buck Converters," IEEE Applied Power Electronics Conference and Exposition, APEC 2004, pp. 1289-1293 vol.2.
[19] P. Zumel, O. Garcia, J. Cobos, J. Uceda,"Magnetic integration for interleaved converters", Applied Power Electronics Conference, 2003.

[20] P. Zumel, O. Garcia, J. A. Cobos and J. Uceda, "Tight magnetic coupling in multiphase interleaved converters based on simple transformers," IEEE Applied Power Electronics Conference, 2005.

[21] T. Schmid, A. Ikriannikov, "Magnetically Coupled Buck Converters," IEEE Energy Conversion Congress and Exposition, pp. 4948-4954.

[22] A.V. Ledenev, G.G. Gurov, and R.M. Porter, "Multiple Power Converter System using Combining Transformers," U.S. Patent 6,545,450, April 8, 2003.

[23] A. Ikriannikov, "The Benefits of the Coupled Inductor Technology," Tutorial 5997, Maxim Integrated, 2014.

[24] M. Ursino, S. Saggini, S. Jiang and C. Nan, "High Density 48Vto-PoL VRM with Hybrid Pre-Regulator and Fixed-Ratio Buck," IEEE Applied Power Electronics Conference, 2020. 